

Polibotánica

ISSN electrónico: 2395-9525

polibotanica@gmail.com

Instituto Politécnico Nacional

México

http://www.polibotanica.mx

\title{
DISTRIBUCIÓN GEOGRÁFICA DE ESPECIES DE Euphorbia sect. Poinsettia CON POSIBLE USO ORNAMENTAL EN MÉXICO
}

\section{GEOGRAPHIC DISTRIBUTION OF SPECIES OF Euphorbia sect. Poinsettia WITH POTENTIAL ORNAMENTAL USE IN MEXICO}

M. L. Pérez-Nicolás, M.T. Colinas-León, M.C. Gómez-Alanis, C. Flores-Espinosa, I. AliaTejacal y M. G. Peña-Ortega

DISTRIBUCIÓN GEOGRÁFICA DE ESPECIES DE Euphorbia sect. Poinsettia CON POSIBLE USO ORNAMENTAL EN MÉXICO

GEOGRAPHIC DISTRIBUTION OF SPECIES OF Euphorbia sect. Poinsettia WITH POTENTIAL ORNAMENTAL USE IN MEXICO

\section{POLIBeTÁNICA}

Instituto Politécnico Nacional
Núm. 50: 165-189 México. Agosto 2020

DOI: $10.18387 /$ polibotanica.50.12

(c) (i) Este es un artículo de acceso abierto bajo la licencia Creative Commons 4.0 Atribución-No Comercial (CC BY-NC 4.0 Internacional). 


\section{DISTRIBUCIÓN GEOGRÁFICA DE ESPECIES DE Euphorbia sect. Poinsettia} CON POSIBLE USO ORNAMENTAL EN MÉXICO

\section{GEOGRAPHIC DISTRIBUTION OF SPECIES OF Euphorbia sect. Poinsettia WITH POTENTIAL ORNAMENTAL USE IN MEXICO}



\author{
M. L. Pérez-Nicolás \\ M.T. Colinas-León / lozcol@gmail.com \\ Departamento de Fitotecnia. Universidad Autónoma Chapingo. Km 38.5 Carr. \\ México-Texcoco. Chapingo, Estado de México. CP 56230. Tel. 5959521500.
}

M.C. Gómez-Alanis

División de nanotecnología. Universidad Politécnica del Valle de México. Av. Mexiquense s/n esquina Av. Universidad Politécnica, Col. Villa Esmeralda, Tultitlán, C.P. 54910, Estado de México. Tel. 5550626460.

C. Flores-Espinosa Departamento de Fitotecnia. Universidad Autónoma Chapingo. Km 38.5 Carr. México-Texcoco. Chapingo, Estado de México. CP 56230. Tel. 5959521500.

I. Alia-Tejacal

Facultad de Ciencias Agropecuarias, Universidad Autónoma del Estado de Morelos. Av. Universidad 1001, Cuernavaca, Morelos. CP 62210. Tel. 7771345402.

M. G. Peña-Ortega

Departamento de Fitotecnia. Universidad Autónoma Chapingo. Km 38.5 Carr. México-Texcoco. Chapingo, Estado de México. CP 56230. Tel. 5959521500.

RESUMEN: En los últimos años se han enlistado especies del género Euphorbia con potencial de uso ornamental como respuesta a la demanda de los floricultores de contar con variedades mexicanas, ya que la mayoría de las variedades ornamentales que se comercializan de este género provienen de otros países. Este trabajo tuvo como objetivo determinar la distribución geográfica de diez especies de Euphorbia sect. Poinsettia subsect. Stormiae y evaluar su potencial para ser utilizadas como plantas de ornato. Se elaboró una matriz de datos con registros de herbarios y recolectas en campo, se hicieron mapas de distribución y se analizó con base en hábitos de crecimiento, tipos de clima y tipos de suelo a través de Sistemas de Información Geográfica. Se definió el potencial ornamental con base en valores estéticos y se documentó la fase del proceso de generación de nuevos cultivos hortícolas en que se encuentran. Los estados donde se localizó el mayor número de especies fueron Jalisco (6), Nayarit (5) y Sinaloa (5). Con base en la distribución geográfica se establecieron dos grupos: hierbas anuales con amplia distribución y hierbas perennes o arbustos con distribución restringida. Las especies se localizan principalmente en climas cálidos y templados subhúmedos, en suelos de tipo litosol, regosol y cambisol. Los valores estéticos que se presentaron en su mayoría corresponden a brácteas atractivas. Euphorbia cornastra, E. pulcherrima y E. strigosa han pasado la Fase I en el proceso de investigación hacia nuevos cultivos ornamentales y se encuentran en la Fase II. Las especies se distribuyen principalmente en los estados ubicados en la vertiente del Pacífico y la mayoría son endémicas de México. El potencial ornamental de las especies se definió tanto por sus valores estéticos como por su comportamiento al ser cultivadas.

Palabras clave: Euphorbiaceae, endemismo, SIG, subgénero Chamaesyce. 


\begin{abstract}
In recent years, species of the genus Euphorbia with potential for ornamental use have been proposed in response to the demand for flower growers to have Mexican varieties since most of the ornamental varieties that are marketed come from abroad. This work is aimed to determine the distribution of Mexican species of Euphorbia subsect. Stormiae and evaluate if they can be used as ornamental plants. A data matrix was prepared with herbarium specimens and field collections, distribution maps were prepared and analyzed based on growth habit, climate types and soil types with Geographic Information Systems. The potential for use of a species was defined based on aesthetic values, and it was documented in which phase of the process of generating new horticultural crops they are. The states where the highest number of species are located are: Jalisco (6), Nayarit (5) and Sinaloa (5). Based on their geographic distribution, two groups were established: annual herbs with a wide distribution and perennial herbs or shrubs with a restricted distribution. The species mainly in warm and termperate subhumid climates and on lithosol, regosol, and cambisol soils. The aesthetic values that were presented mostly correspond to showy bracts. Euphorbia cornastra, E. pulcherrima and E. strigosa are in phase II of the research process towards new crops. The species are distributed mainly in the states located on the Pacific slope of Mexico and most of them are endemic. The potential for ornamental use of a species is defined both by its aesthetic value and by their behaviour when cultivated.
\end{abstract}

Key words: Euphorbiaceae, endemism, SIG, subgenus Chamaesyce.

\title{
INTRODUCCIÓN
}

La familia Euphorbiaceae sensu lato es una de las familias más diversas a nivel mundial, incluye alrededor de 8000 especies (Webster, 1994). En recientes clasificaciones taxonómicas se propone su división en cinco familias: Phyllanthaceae, Picrodendraceae, Peraceae, Putranjivaceae y Euphorbiaceae sensu stricto (APG IV, 2016). En México se han reportado diferentes números de especies que la constituyen: 782 (Steinmann, 2002), 826 (MartínezGordillo et al., 2002); 710 (Martínez et al., 2008) y recientemente Villaseñor (2016) señaló 714 especies, con lo que esta familia ocuparía el quinto lugar en diversidad después de Asteraceae, Fabaceae, Orchidaceae y Poaceae.

Es una familia con varias especies útiles y uno de los usos más importantes es el ornamental, que hace referencia a las plantas que se utilizan con propósitos decorativos y que responden a valores estéticos asignados por personas en diferentes contextos culturales (Hurrel, 2016). Euphorbia pulcherrima Willd. ex Klotzsch (nochebuena) es la especie ornamental más importante y comercializada en todo el mundo y se encuentra dentro de uno de los géneros con mayor número de especies. El género Euphorbia en nuestro país contiene 241 especies (Steinmann, 2002; Martínez et al., 2008) y la cifra más reciente es de 245 especies (Villaseñor, 2016), con un alto porcentaje de endemismo. Otras especies ornamentales del mismo género comercializadas son E. pteroneura A. Berger, E. leucocephala Lotsy, E. milii Dess Moul, E. graminea Jacq. y un gran número de suculentas como E. trigona Haw. y E. obesa Hook. f. (Steinmann, 2002).

En nuestro país, los productores de ornamentales mencionan que existe la necesidad de contar con variedades de origen mexicano que sean más resistentes y que se adapten a las condiciones climáticas de cada región para con ello reducir costos de producción, ya que actualmente las variedades que se comercializan provienen del extranjero por lo que se requiere pagar derechos de obtentor (Canul-Ku et al., 2010). Diversos autores han enlistado especies con potencial ornamental, integrando plantas que se encuentran en catálogos de horticultores de diferentes países, plantas que se usan a nivel regional o local y plantas que no se usan pero que por sus características estéticas se les puede dar dicho uso (Chimal \& Corona, 2003; GuadarramaMartínez et al., 2012; Corona \& Chimal, 2006; Pérez-Nicolás \& Fernández-Nava, 2007; Rendón-Correa \& Fernández-Nava, 2007; Rzedowski, 1995). En los listados se encuentran 13 
especies del género Euphorbia, incluyendo a la nochebuena con potencial de ser utilizadas en México.

Los programas de mejoramiento y la investigación de nuevos cultivos ornamentales a nivel mundial están enfocados en tres áreas: la primera, en investigar sobre especies ornamentales bien establecidas, buscando nuevas formas y colores; la segunda, en encontrar nuevos usos en especies conocidas, por ejemplo, si una planta es usada como planta en maceta estudiar si se puede utilizar como flor de corte y la tercera en estudiar especies poco conocidas sobre las cuales existe poca o ninguna información (Armitage, 1986, 1987; Le Duc \& Albrecht, 1996). En México dentro de la primera área se encuentran las dalias (Dahlia pinnata Cav.), el cempasúchil (Tagetes erecta L.) y la nochebuena (Euphorbia pulcherrima). Por lo que, se ha utilizado material vegetal de nochebuenas de traspatio y cultivadas para generar variedades de nochebuena mexicanas. A la fecha se han registrado tres variedades: Rubí, Alondra y Juanita, las cuales, aunque ya se encuentran entre los productores aún no se comercializan (Canul-Ku et al., 2018, 2019; Canul Ku et al., 2017). En la tercera área se encuentra Euphorbia leucocephala (pascuita) y E. pulcherrima (nochebuena de sol o de exterior) que son comercializadas a nivel regional y de las cuales existe escasa información (Colinas-León et al., 2015; Galindo-García et al., 2012, 2019; Martínez-Villegas et al., 2015). Sin embargo, a la fecha hay pocas líneas de investigación sobre especies silvestres con potencial de llegar a ser plantas ornamentales. Por lo anterior, para comenzar a investigar sobre las especies del género Euphorbia con potencial ornamental se decidió seleccionar especies cercanas a la nochebuena, que ha sido incluida dentro de diversas propuestas taxonómicas que hacen referencia a Poinsettia.

Poinsettia fue propuesto por primera vez como género por Graham (1836). Baillon (1987) propuso que se tratara como una sección del género Euphorbia. Bossier (1862) dividió al género Euphorbia en 27 secciones, la sección 15 denominada Poinsettia contenía 11 especies. House (1924) consideró tratar como Subgénero Poinsettia. Dressler (1962) lo trató como un género aparte de Euphorbia, aunque esta propuesta no ha sido aceptada. Mayfield (1997) realizó un estudio sistemático del Subgénero Poinsettia, en el cual incluyo 24 especies (Apéndice 1).

Las clasificaciones más recientes basadas en datos filogenéticos moleculares ubican a la nochebuena dentro del subgénero Chamaesyce sect. Poinsettia (Horn et al., 2012; Yang et al., 2012). Está sección se divide en cuatro subsecciones: subsect. Lacerae, subsect. Erianthae, subsect. Exstipulatae y subsect. Stormieae. Las tres primeras tienen algún tipo de apéndice en la glándula involucral, mientras que la última subsección no presenta apéndices, sólo Euphorbia chersonesa y E. cornastra presentan un apéndice rudimentario y obsoleto (Yang et al., 2012).

Euphorbia subsect. Stormiae Croizat, incluye a hierbas anuales o perennes, arbustos o pequeños árboles, hojas opuestas y a menudo se alternan en la sección media de la planta y luego son opuestas o en espiral en las inflorescencias, lineares a panduradas y generalmente dentadas; brácteas coloreadas y brillantes, ciatios en cimas terminales, generalmente densas, a veces cimas monocasiales; glándulas involucrales en forma de copa de 1-5 (-8), estilos tres libres o connados en la base, bífidos, rara vez enteros o con sólo el ápice bifurcado, ovario y cápsula glabros o pubescentes de 3 lóculos, semillas ovoides, cuadrangulares o algo redondeadas en sección transversal, gruesamente tuberculadas, ecarunculadas o carúnculas de varias formas. Se distribuyen en el nuevo mundo desde Canadá hasta Argentina, con un centro distribución en México, crecen en una amplia variedad de hábitats desde matorrales desérticos hasta bosques húmedos de montaña, desde el nivel de mar hasta 2,700 m (Yang et al., 2012), por lo que se considera que, dentro de este grupo, se pueden encontrar especies que tienen el potencial de ser cultivadas como plantas de ornato.

Las especies incluidas en el presente estudio se ubican dentro de esta subsección y son: Euphorbia heterophylla L., E. cyathophora Murray, E. pulcherrima Willd. ex Klotzsch, E. pumicicola Huft, E. hormorrhiza Radcl.-Sm., E. restiaceae Benth., E. cornastra (Dressler) 
Radcl.-Sm., E. colorata Engelm., E. strigosa Hook. \& Arn., E. radians var. radians Benth. y E. radians var. stormiae (Croizat) Rzed. \& Calderón. Se han realizado pocos trabajos sobre ellas, entre los que se encuentran: evaluación del potencial ornamental de E. cornastra realizado en Estados Unidos (Le Duc \& Albrecht, 1996) y estudios anatómicos en México (HernándezCarrillo, 2014). Propuesta de cultivo in vitro de E. cyathophora (Rodríguez-Elizalde et al., 2019), ensayos de germinación de E. heterophylla (Cerón-Venegas, 2006) y caracterización de semillas, cultivo in vitro y distribución geográfica de E. strigosa (Cázarez-Prado, 2013; CerónVenegas, 2006; E. F. Valdez-Hernández et al., 2018; Edna F. Valdez-Hernández et al., 2017). Por último, caracterizaciones morfológicas, diversidad genética y distribución geográfica de $E$. pulcherrima (Cerón-Venegas, 2006; Flores-Cabrera, 2014; González-Fierro, 2007; TrejoHernández et al., 2015; Trejo et al., 2012). Por consiguiente, este estudio tuvo como objetivo definir la distribución geográfica de especies de Euphorbia sect. Poinsettia subsect. Stormiae, documentar aspectos de su morfología y hábitat, así como analizar su potencial para ser usadas como plantas de ornato, bajo el supuesto de que al ser especies cercanas a la nochebuena comparten características morfológicas con dicha especie y por tanto pueden presentar y adaptarse a condiciones de cultivo.

\section{MATERIAL Y MÉTODOS}

\section{Distribución geográfica}

Base de datos: La consulta de ejemplares se realizó en dos etapas; la primera entre los años 2009-2012 en 21 herbarios (BCMEX, CEDESU, CHAP, CHAPA, JES, CHIP, ECO-SC-H, CICY, CIMI, CIQR, GUADA, UAGRO, HCIB, ENCB, HUMO, IEB, MEXU, UADY, UCAM, UJAT, XAL). En cada herbario se corroboró la identificación de especies y se tomaron fotografías. Con la información obtenida se construyó una matriz de datos que contiene: especie, nombres comunes, país, estado, municipio, localidad, latitud, longitud, altitud, hábitat, observaciones, usos, nombre y número del colector, fecha de colecta, nombre del determinador y herbario donde se localiza el ejemplar.

La segunda etapa se realizó entre los años 2018-2019, donde se consultaron bases de datos de colecciones nacionales e internacionales disponibles en línea y con acceso libre: Red Mundial de Información sobre Biodiversidad (REMIB, 2015), Global Biodiversity Information Facility (GBIF, 2018) y el portal de Datos Abiertos de la UNAM (2019). Se visitaron nuevamente los ejemplares de las colecciones CHAP, CHAPA y JES debido a que sus bases digitales no están disponibles en línea. Se realizó una revisión nomenclatural de las especies en las bases de datos de Missouri Botanical Garden (Tropicos, 2018) y en International Plant Names Index (IPNI, 2019). Los datos obtenidos se adicionaron a la base antes mencionada y se eliminaron registros que contenían localidades repetidas para la misma especie.

Elaboración de mapas: El proceso de georreferenciación estuvo constituido, en orden secuencial, por la normalización de la información de la base de datos; validación de las coordenadas geográficas y en los registros de herbario que no contaban con coordenadas geográficas fueron georreferenciados siguiendo la metodología propuesta por la Comisión Nacional para el Conocimiento y Uso de la Biodiversidad (CONABIO, 2008). Las coordenadas geográficas fueron convertidas a grados decimales y se integró la información para su representación en un mapa. Los mapas se elaboraron en el sistema de Información Geográfica ArcMap 10.8 (ESRI, 2019). Posteriormente se hizo una clasificación por hábito de crecimiento en hierbas anuales, hierbas perennes y arbustos, se elaboraron mapas a los cuales se sobrepusieron versiones digitales de los mapas de tipos de climas (INEGI, 2008) y tipos de suelo (INEGI, 2014).

Validación en campo: Con base en los mapas de distribución geográfica, se seleccionaron sitios con mayor número de registros de colecta y con la finalidad de ubicar las poblaciones de las especies se realizaron exploraciones en los estados de Sinaloa, Nayarit, Jalisco, Durango, 
Chihuahua, Guerrero, Oaxaca, Chiapas, Morelos, Yucatán, Quintana Roo y Campeche. En cada sitio donde se observó alguna población, se georreferenció, se tomaron datos de altitud y tipo de vegetación y se colectaron ejemplares de herbario que se depositaron en el Herbario JES. Los sitios de colecta se incluyeron en los mapas de distribución elaborados para cada especie.

\section{Potencial de uso ornamental}

En los sitios donde se realizaron los recorridos antes mencionados se hizo un muestreo dirigido, se observaron los individuos de cada población y se eligieron aquéllos con características atractivas como brácteas grandes, de colores brillantes, también se tomó en cuenta que los individuos estuvieran sanos, teniendo precaución de no seleccionar más del 15\% de cada población. Se colectaron estacas de plantas leñosas y semillas de leñosas y hierbas, esto dependió de la etapa en desarrollo en que se encontraban las plantas. Las estacas se colocaron en papel periódico húmedo en bolsas de plástico hasta su establecimiento en el Banco de Germoplasma de la Universidad Autónoma Chapingo (UACh) y las semillas en bolsas de papel hasta depositarlas en el Banco de Germoplasma de la UACh.

Se definió el potencial ornamental con base en valores estéticos (Pérez-Nicolás \& FernándezNava, 2007; Rendón-Correa \& Fernández-Nava, 2007), es decir, a partir de aspectos decorativos como arquitectura de la planta, flores, frutos, color, olor, formas, texturas o la combinación de varios elementos (Hurrel, 2016; Li \& Zhou, 2005). Además, se documentó su comportamiento al ser cultivadas y las actividades realizadas en el banco de germoplasma. Por último, se determinó en qué fase del proceso de desarrollo de nuevos cultivos se encuentran que hace referencia a su establecimiento bajo cultivo, propagación, producción y comercialización (fig. 1).

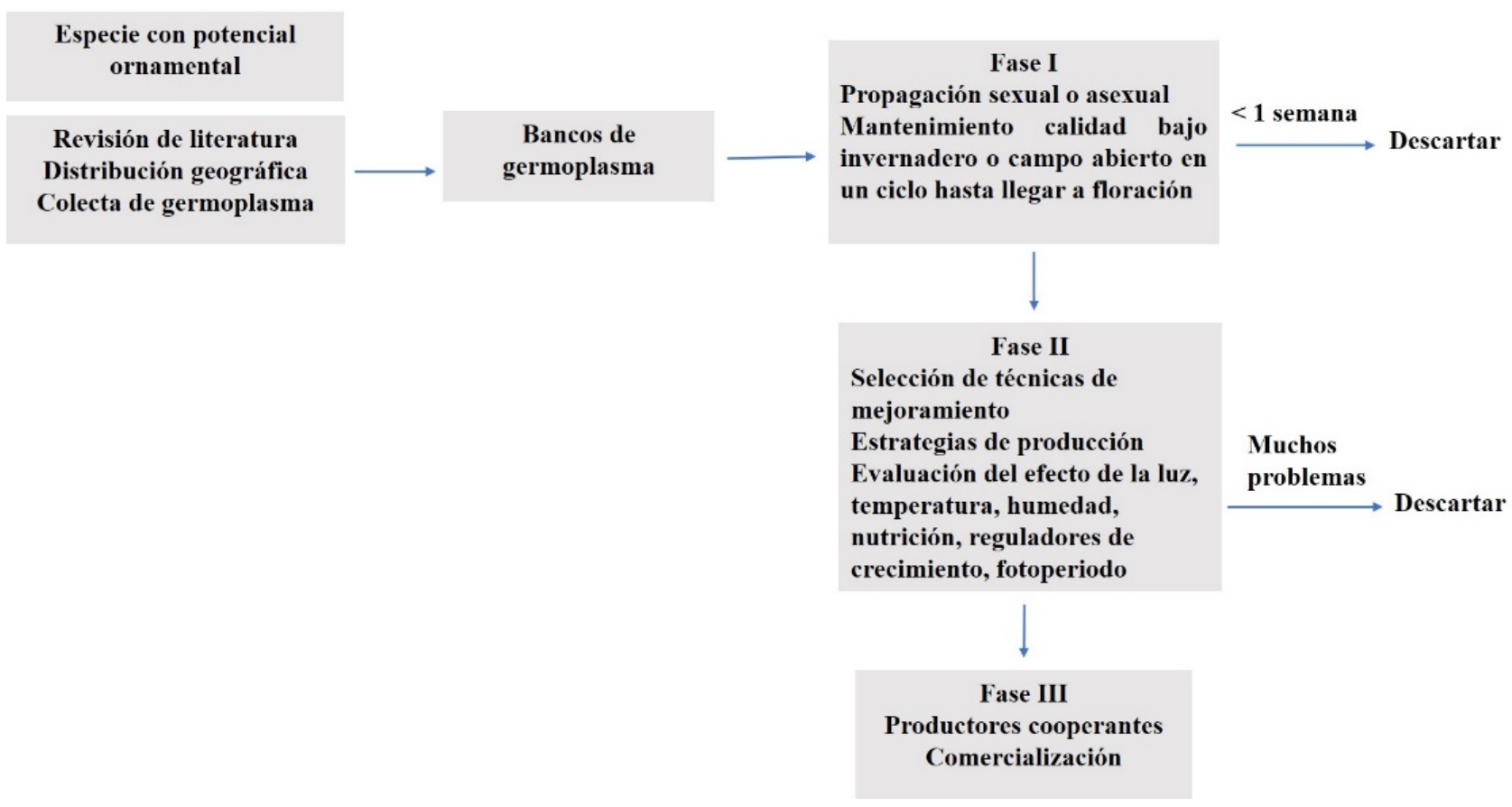

Fig. 1. Fases a considerar en la búsqueda de nuevos cultivos para uso ornamental. Modificado de Armitage (1986). 


\section{RESULTADOS}

\section{Distribución geográfica}

Se revisaron 1,703 ejemplares en los diferentes herbarios, de los cuales en 1,540 se pudo corroborar la identificación. No obstante, en una búsqueda actual en las bases de datos digitales se obtuvieron 2,471 ejemplares. A pesar de que algunos ejemplares revisados en físico se encuentran en las bases de datos digitales, sin embargo, en estas bases no se localizaron ejemplares de herbarios como CHAP, JES y CHAPA. En contraste, en las bases de datos digitales de los herbarios ARIZ, MO, LL, TEX, DUKE se localizan ejemplares que no se revisaron en físico. Se registraron diversos nombres comunes y diferentes usos como el medicinal, comestible, forrajero y ornamental (Apéndice 2). Los estados con mayor número de registros con base en la revisión de ejemplares en físico fueron Jalisco (213), Veracruz (152) y Chiapas (131). Los estados donde se localizan el mayor número de especies de Euphorbia subsect. Stormiae fueron Jalisco (6) y un taxa intraespecífico, Nayarit (5), Sinaloa (5), Chihuahua (5) y Guerrero (5), por lo que la mayor diversidad se localiza en estados ubicados en la vertiente del Pacífico.

Las especies estudiadas se pueden dividir en dos grupos de acuerdo a su distribución geográfica. El primero incluye especies con distribución amplia, que se localizan en varios estados del país y en otros países americanos, se ubican en selvas bajas caducifolias, bosques de encino, matorrales y vegetación secundaria y que habitan en un amplio rango altitudinal desde el nivel del mar hasta los $2600 \mathrm{~m}$ (Apéndice 3). Euphorbia cyathophora se distribuye en Estados Unidos, Centroamérica y Sudamérica. En México se localiza en la mayoría de los estados del país, excepto en la CDMX, Aguascalientes, Guanajuato y Tlaxcala. En la península de Yucatán se encuentra el mayor número de colectas y hay un número considerable en los estados de Chiapas, Oaxaca, Guerrero y Coahuila (fig. 2). En el estado de Sinaloa sólo se encontró un ejemplar de herbario colectado en Mazatlán. Huft (1984) reporta su ubicación en Baja California, pero en esta búsqueda no se encontraron ejemplares en ese estado. Mayfield (1997) examinó ejemplares de Costa Rica, Honduras, Panamá, Argentina, Colombia, Ecuador, Paraguay, Venezuela, Bermuda, Bahamas y Antillas. En los recorridos efectuados en campo se ubicaron plantas creciendo a orillas de caminos o en sitios perturbados, teniendo un comportamiento de maleza.

Euphorbia heterophylla, presenta una distribución amplia, se localiza en Estados Unidos, Centroamérica y Sudamérica. En México se encuentra en la mayoría de los estados excepto en Coahuila y CDMX. Esta especie se ha colectado principalmente en el centro y sur del país, el mayor número de registros se encuentra en Chiapas (fig. 3). Huft (1984) reporta su ubicación en Baja California. Durante las exploraciones realizadas en campo se observaron gran número de plantas en cultivos, orillas de camino y en sitios perturbados y vegetación secundaria derivada de distintos tipos de vegetación, principalmente selvas bajas caducifolias. Adicionalmente se observó que animales domésticos se alimentan de esta especie.

Euphorbia pulcherrima se distribuye en Guatemala y en México se encuentra desde Sinaloa hasta Chiapas, también hay poblaciones en el estado de Morelos (fig. 4); sin embargo, hay ejemplares de herbario en la mayoría de los estados del país y también de Costa Rica, Nicaragua y Panamá, aunque se trata de materiales cultivados o con algún grado de manejo. Exploraciones en campo permitieron localizar poblaciones de E. pulcherrima, tres ubicadas en Guerrero, tres en Nayarit, una en Morelos, una en Chiapas, cuatro en Oaxaca y una en Sinaloa. Dichas poblaciones están integradas por un gran número de plantas y se ubican principalmente en cañadas de difícil acceso. Algunas de ellas presentan deterioro debido a actividades del ser humano. Las poblaciones con menos disturbio se localizan en lugares apartados de zonas habitadas. Se observó que, en muchos lugares, principalmente en poblados cercanos a donde se encuentran localizadas las poblaciones silvestres de esta especie, las personas se llevan estacas para cultivarlas, por lo que es posible observar la especie en huertas, cultivos y orillas de camino. En ocasiones estas plantas se abandonan y éstas se propagan por sí mismas originando 
manchones de plantas que pueden confundirse con poblaciones silvestres. Adicionalmente se documentó una población en Oaxaca a partir de la cual se extraen nochebuenas silvestres que son comercializadas como flor de corte en los mercados cercanos a la comunidad.

Euphorbia radians se distribuye en Estados Unidos y principalmente en estados del centro y norte de México, también se ubica en el estado de Oaxaca (fig. 5). El mayor número de colectas se ha hecho en el Estado de México, mientras que Euphorbia radians var. stormiae se ha colectado en tres estados: Michoacán, Jalisco y Estado de México.

El segundo grupo está conformado por especies con distribución restringida y endémicas. La mayoría se distribuyen en la vertiente del Pacífico, excepto, E. hormorrhiza que se localiza en la vertiente del Golfo de México. Se localizan principalmente en bosque de encino, bosque de pino-encino, selva baja caducifolia y en zonas de transición entre estos tipos de vegetación, en un rango altitudinal de 60 a 2400 m (Apéndice 3). Euphorbia colorata se localiza en el noroeste de México en Sonora, Chihuahua, Durango, Sinaloa y los límites de Durango y Zacatecas. En el portal de datos abierto de la UNAM se localizaron dos ejemplares, uno corresponde al estado de Guerrero y el otro al estado de Morelos; sin embargo, como no se revisaron físicamente los ejemplares, no se incluyeron en el mapa (fig. 6).



Fig. 2. Distribución geográfica de Euphorbia cyathophora con base en ejemplares de herbario y colectas realizadas en este trabajo. 


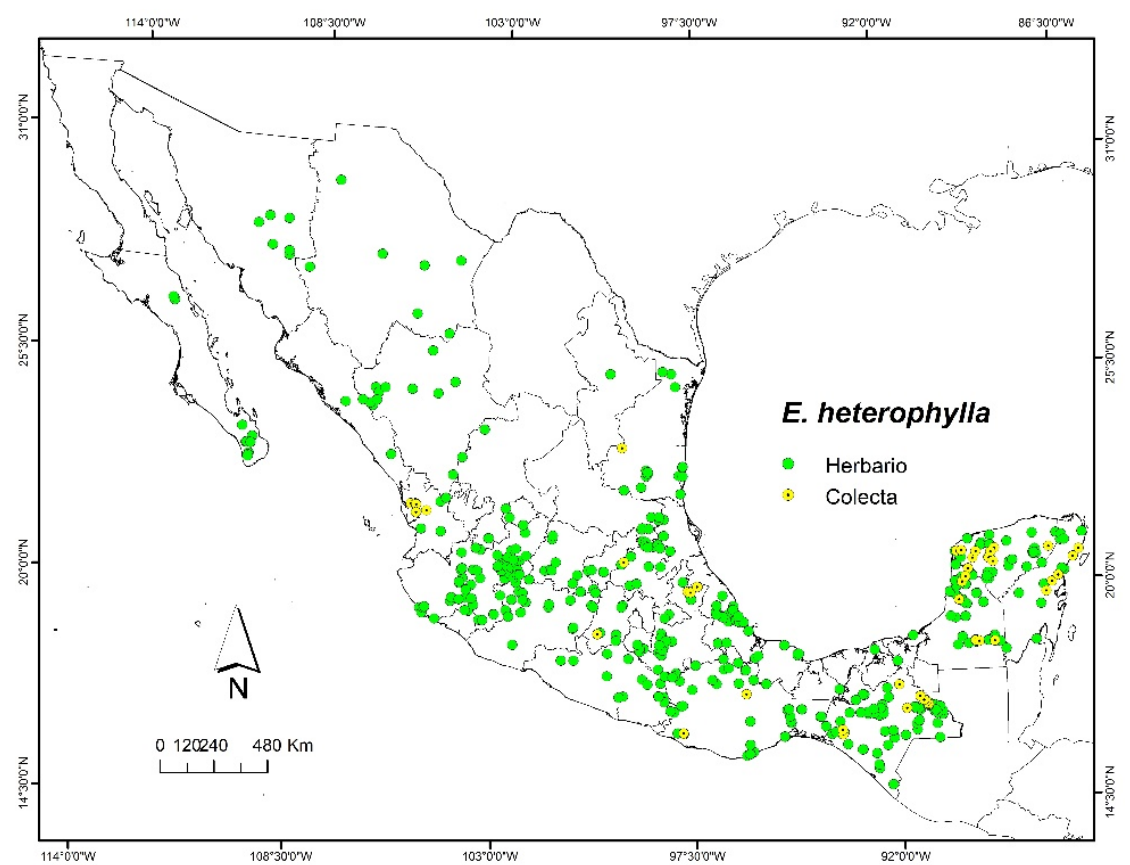

Fig. 3. Distribución geográfica de Euphorbia heterophylla con base en ejemplares de herbario y colectas realizadas en este trabajo.

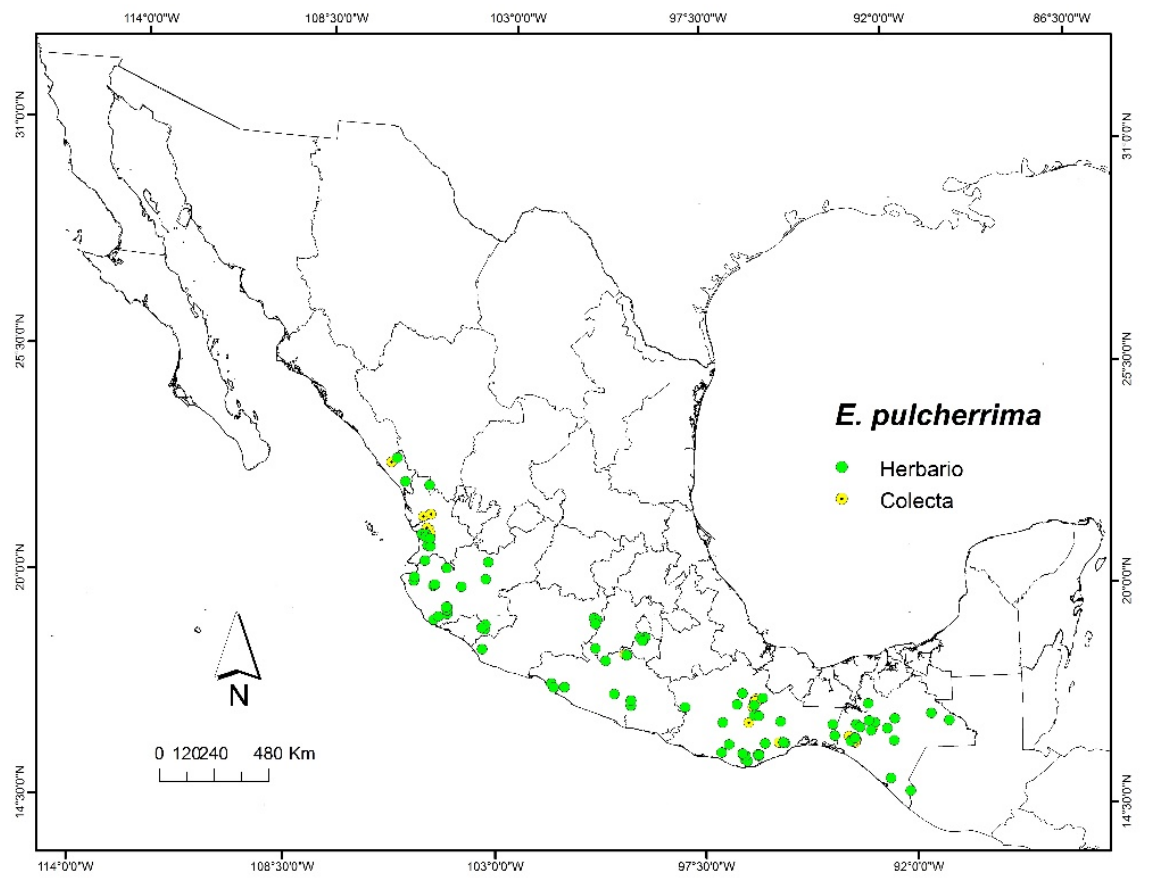

Fig. 4. Distribución geográfica de Euphorbia pulcherrima con base en ejemplares de herbario y colectas realizadas en este trabajo. 


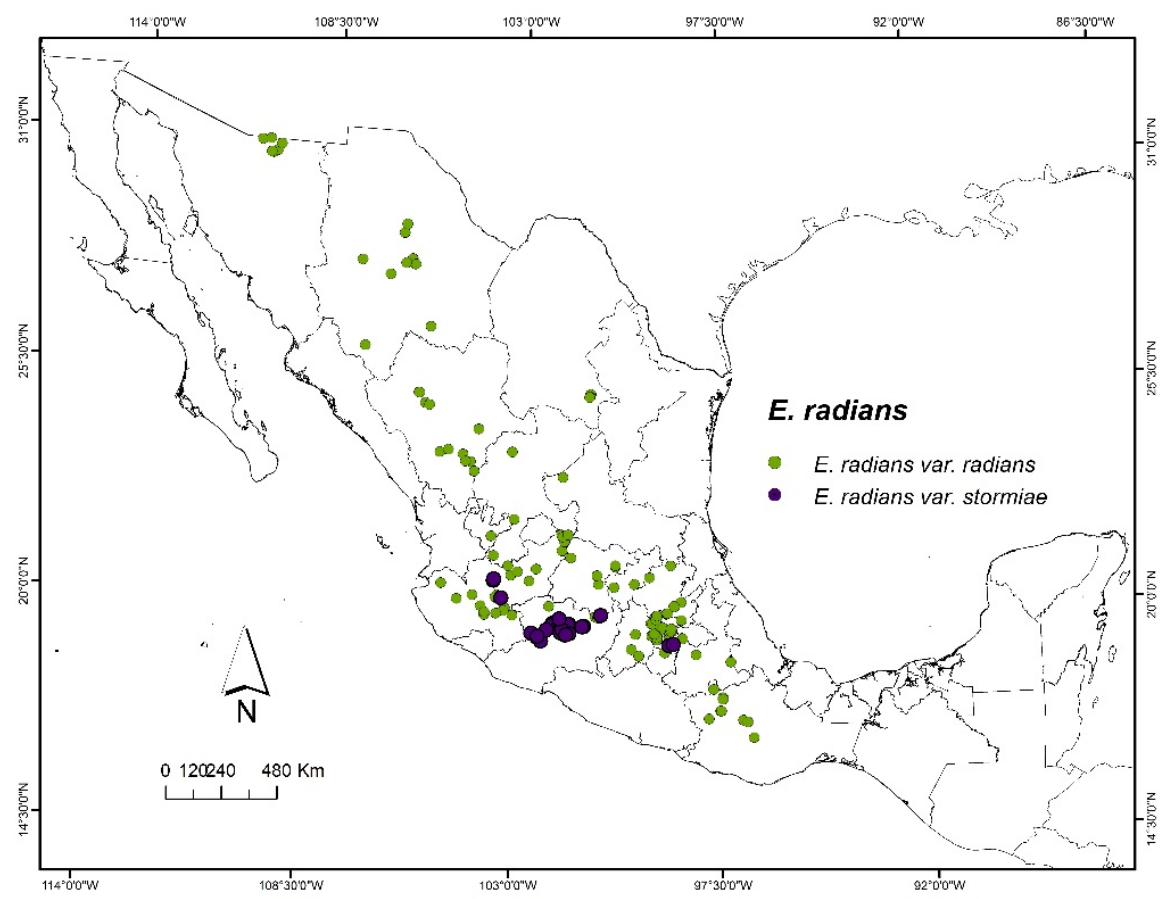

Fig. 5. Distribución geográfica en México de Euphorbia radians y E. radians var. stormiae.

Euphorbia strigosa se distribuye principalmente en el occidente del país, con algunos sitios de colecta en el noroeste, en Colima, Durango, Jalisco, Nayarit, Sonora, Sinaloa, en los límites de Sonora con Chihuahua, de Sinaloa con Chihuahua y de Jalisco con Zacatecas. En los recorridos en campo se encontraron tres poblaciones en Jalisco y dos en Nayarit (fig. 7).

La distribución de Euphorbia restiaceae y E. pumicicola se restringe a dos estados, la primera se ubica en Jalisco y Nayarit, mientras que la segunda se localiza en Baja California Sur y Sonora. La distribución de Euphorbia hormorrhiza y E. cornastra se restringe a un sólo estado, la primera se localiza en Tamaulipas y la segunda en Guerrero. En las exploraciones en campo se visitaron los lugares de colecta registrados en los herbarios y sólo fue posible localizar la población de E. cornastra, con un alto grado de perturbación debido a las actividades realizadas por los pobladores, así como a la construcción de una carretera (figs. 6 y 7).

La información obtenida en el presente estudio permite identificar que existen patrones de distribución definidos. Las especies que son hierbas anuales (Apéndice 4), Euphorbia heterophylla y E. cyathophora se localizan en casi todo el territorio Mexicano, lo cual puede deberse a su fácil propagación y adaptación a diversos hábitats. Crecen principalmente en áreas con climas cálido subhúmedo, templado subhúmedo, cálido húmedo, templado húmedo y en menor proporción en climas seco semicálido, semicálido subhúmedo y muy seco semicálido. Habitan sitios perturbados, orillas de caminos y cultivos, localizados en suelos tipo regosol, vertisol, litosol, rendizina, feozem, acrisol, andasol, xerosol, gleysol y andosol (fig. 8). Euphorbia pumicicola a pesar de ser una hierba anual es una especie de distribución restringida en clima muy seco semicálido, muy seco cálido y templado subhúmedo y crece en suelos tipo regosol, vertisol, litosol y yermosol (fig. 8). 


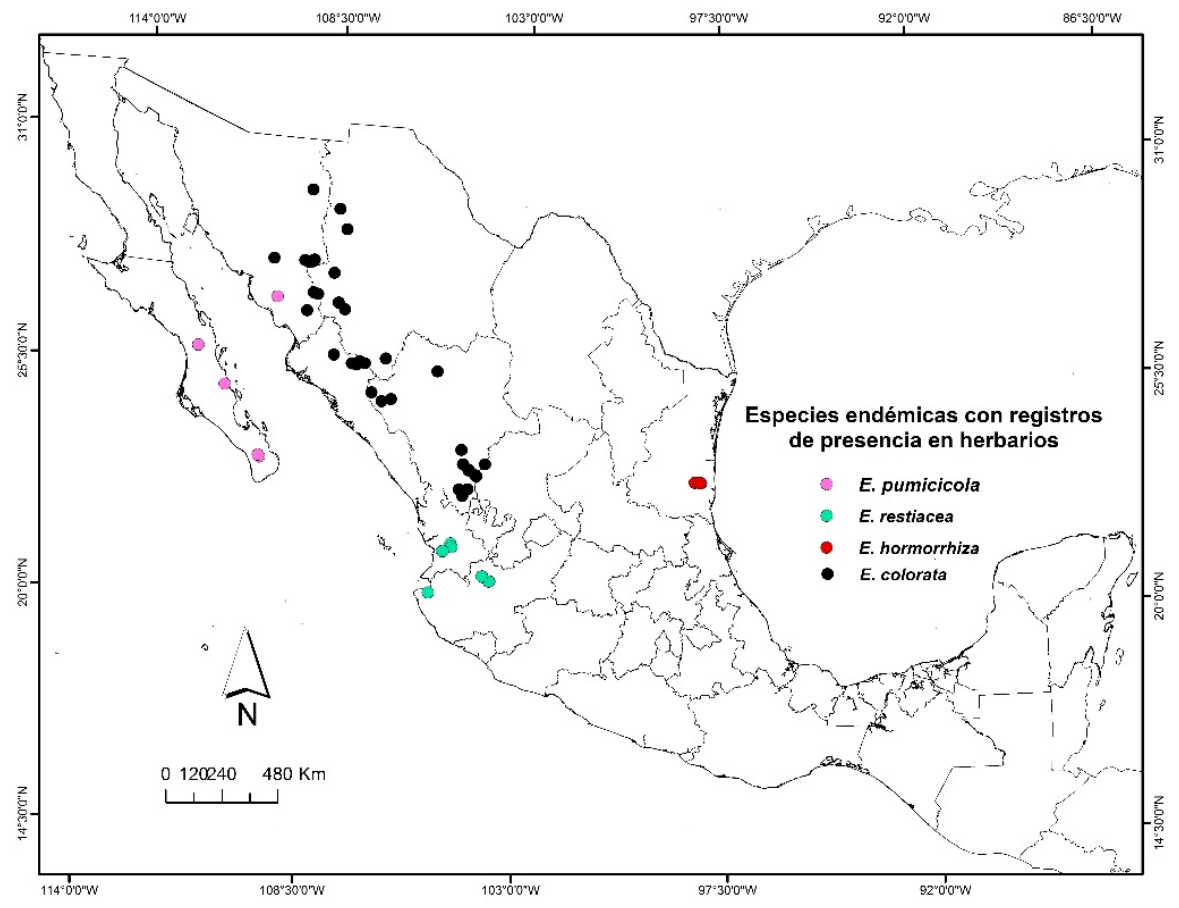

Fig. 6. Distribución geográfica de especies endémicas de México con base en registros de herbarios.



Fig. 7. Distribución geográfica de especies endémicas de México con base en registros de herbarios y colectas en este trabajo. 
Las especies que son hierbas perennes y arbustos (Apéndice 3) se localizan en la vertiente del Pacífico, en climas cálidos húmedos y templados subhúmedos, crecen en suelos de tipo regosol, litosol, cambisol, feozem y en menor proporción en suelos tipo acrisol, luvisol y andosol. $E$. radians es una hierba perenne geófita que se distribuye en su mayoría en climas templado subhúmedo, semiseco templado y semicálido húmedo y se desarrolla en los tipos de suelos consignados para las especies anteriores, así como en yermosol, xerosol y planosol (fig. 9). De manera general, estas especies crecen en suelos con abundante materia orgánica, bien drenados, arenosos, superficiales o poco profundos y en climas cálidos y templados subhúmedos.

\section{Potencial ornamental}

Las especies que se colectaron y se establecieron en las colecciones bajo invernadero fueron Euphorbia cornastra, E. cyathophora, E. heterophylla, E. pulcherrima y E. strigosa (fig. 10). Debido a su hábito de crecimiento arbustivo E. cornastra podría ser utilizada como planta de exterior para jardines, huertos, parques, calles y caminos en zonas con sombra. Asimismo, podría ser usada como planta en maceta para interiores. El color blanco de sus brácteas y su arquitectura arbustiva en forma redondeada y erecta son las principales características estéticas sobresalientes. Un rasgo distintivo de esta especie que puede ser aprovechado en la agronomía es que florece en verano, a diferencia de la nochebuena que florece en invierno, lo cual representa una valiosa alternativa para tener una planta muy parecida a la nochebuena pero que florece en otra época del año. Las plantas de E. cornastra, obtenidas a través de estacas cumplieron con la fase I, produjeron flores, mantuvieron su calidad y tuvieron una vida postcosecha de más de una semana; sin embargo, bajo condiciones de cultivo el tamaño de las brácteas disminuyó comparando con lo observado en campo y con el paso del tiempo la mayoría de las plantas murió.

Las plantas que sobrevivieron se propagaron a través de estacas y de ellas se obtuvieron semillas, las cuales se pusieron a germinar. Se observó que la emergencia del hipocótilo ocurre a los ocho días después de la siembra, con porcentajes de emergencia de $80 \%$; no obstante, muchas plántulas murieron y las que sobrevivieron no alcanzaron a llegar a la etapa de floración, por lo cual se suspendió el proceso de producción. Estos resultados indican que esta especie se encuentra en la Fase II o en etapa de propagación, por lo que sería necesario establecer nuevos experimentos enfocados a mejorar las técnicas para la multiplicación de esta especie.

Euphorbia pulcherrima tiene el potencial de ser utilizada como planta de exterior y de interior, su valor estético se encuentra en el color de las brácteas. Las plantas establecidas han pasado la fase I, produjeron flores y mantuvieron su calidad y tuvieron una vida postcosecha de más de una semana. Las semillas emergen a los cuatro o cinco días, con porcentajes de germinación entre 80 y $95 \%$. Estas plantas se han utilizado para hacer cruzas con variedades cultivadas y se han cosechado los frutos obtenidos, los cuales están siendo evaluados. Se encuentra en la fase II enfocada en la propagación y en un proceso de mejoramiento a través de selección de individuos con características deseables.

Euphorbia strigosa tiene potencial de ser usada como planta de exterior tipo césped y también como planta de interior en maceta. Los valores estéticos que presenta son su arquitectura, crece de manera semierecta o decumbente y se extiende adquiriendo una forma esférica y presenta brácteas de tonalidades rojizas. Las plantas han pasado la fase I manteniendo su calidad y floreciendo en un periodo mayor a una semana; sin embargo, su propagación por semilla es difícil, por lo que se han probado distintos métodos de escarificación a pesar de los cuales se han seguido obteniendo bajos porcentajes de germinación de entre 30 y $40 \%$. Por lo tanto, esta especie se encuentra en la fase II, es decir, se está buscando el mejor método de propagación y monitoreando su comportamiento bajo invernadero. 

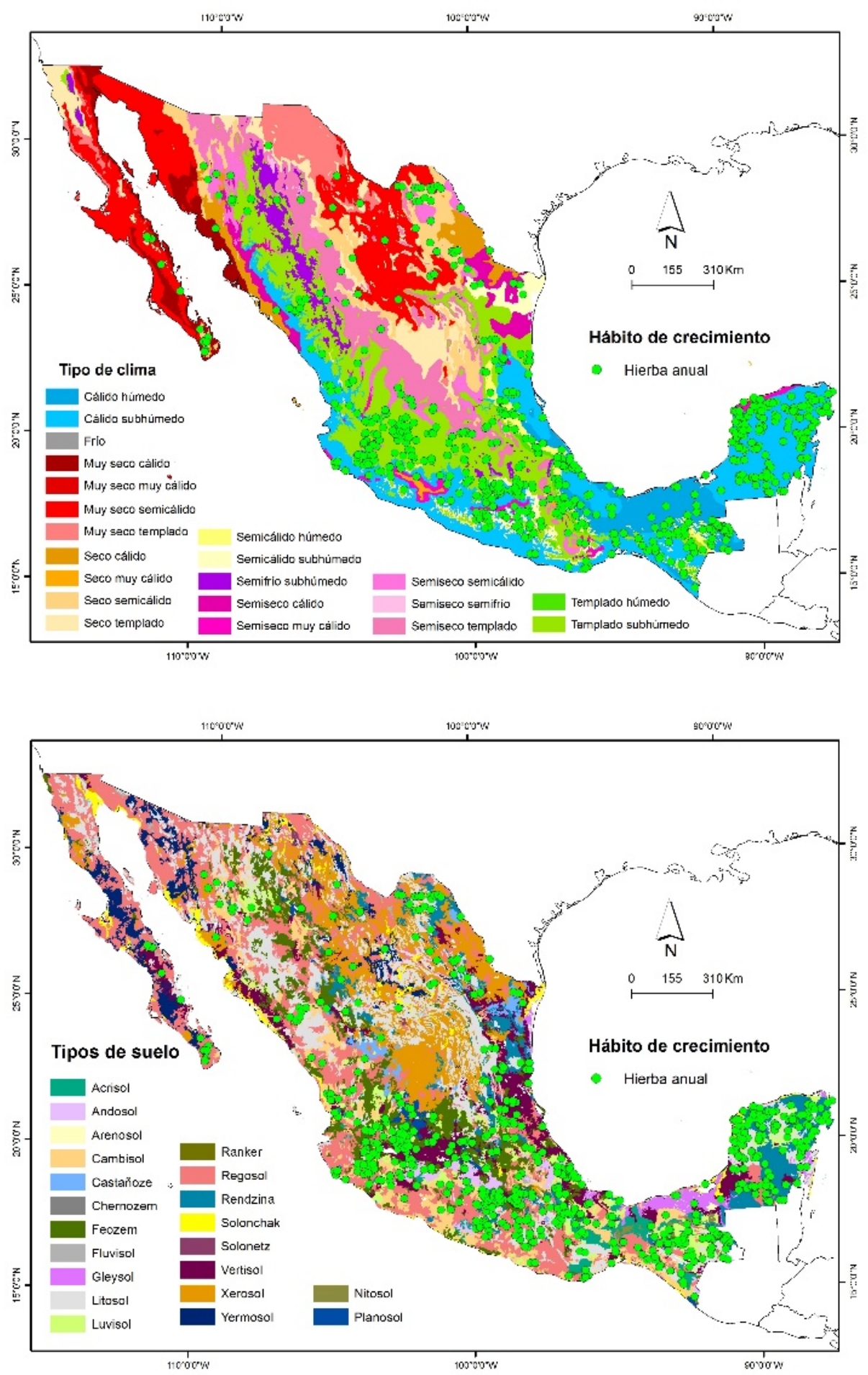

Fig. 8. Distribución geográfica de hierbas anuales con base en el clima y tipo suelo. 

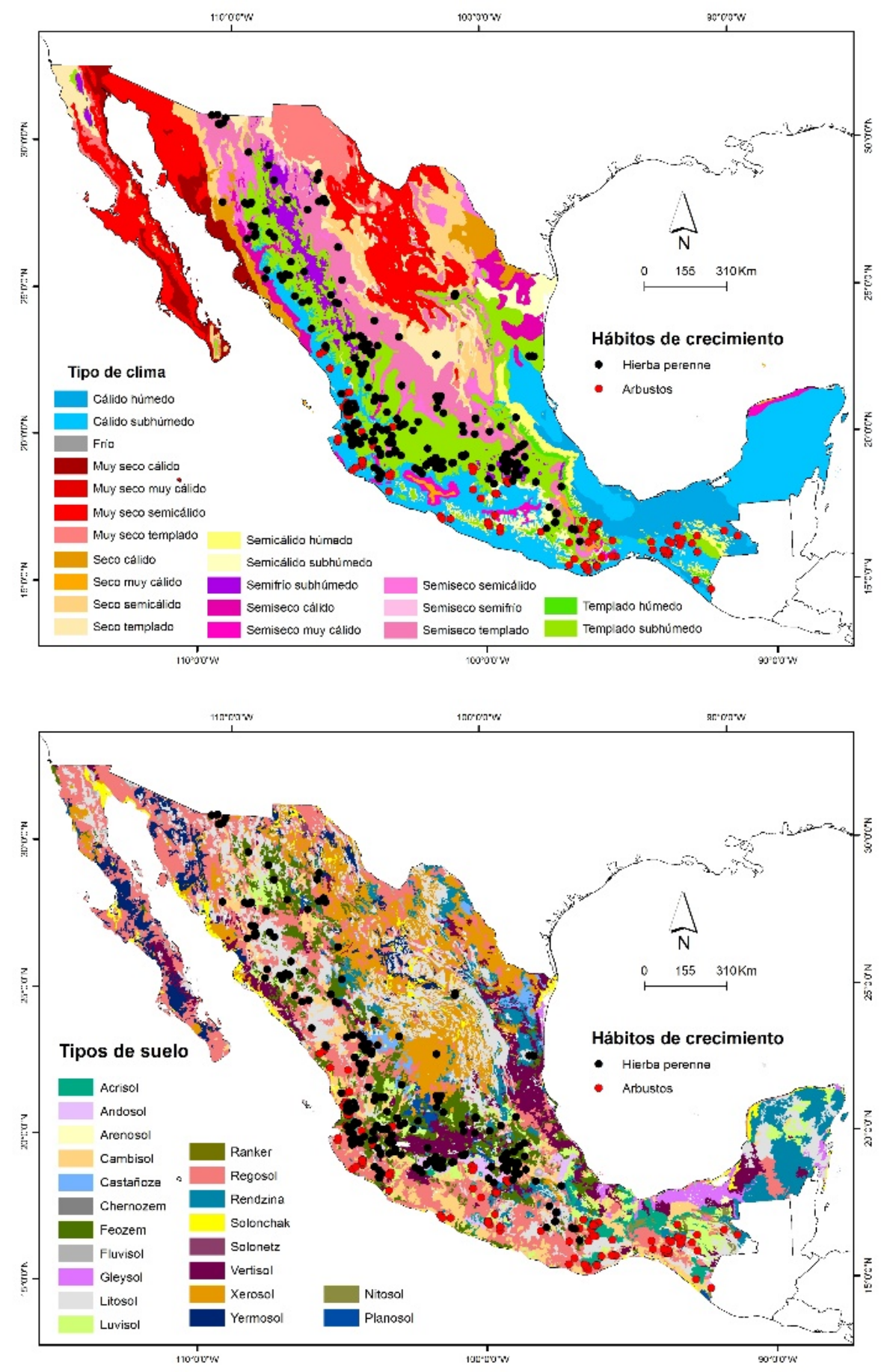

Fig. 9. Distribución geográfica de hierbas perennes y arbustos con base en el tipo de clima.

Euphorbia cyathophora puede ser utilizada como planta de maceta y su valor estético se encuentra en sus brácteas con tonalidades que van del naranja al rojo. Las plantas han pasado la fase I con éxito ya que la propagación por semilla es fácil y se han obtenido varios ciclos de 
floración durante el año manteniendo su calidad bajo invernadero. Euphorbia heterophylla es una especie cercana a la nochebuena, no obstante, carece de valores estéticos. A pesar de ello, las plantas han pasado la fase I debido a que se han obtenido porcentajes de germinación del 90 y $100 \%$. Ambas especies invaden las macetas de otros ejemplares mostrando un comportamiento de maleza, por lo que se han mantenido pocos individuos de las mismas.


A
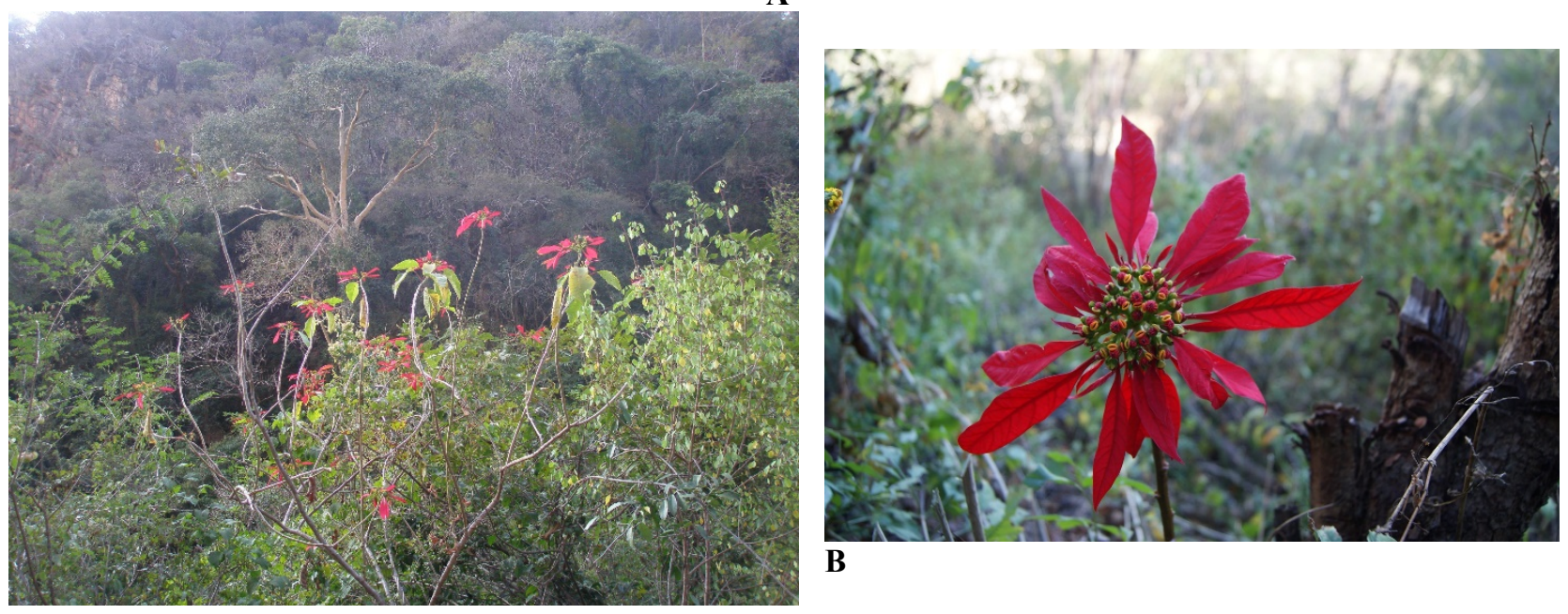

B

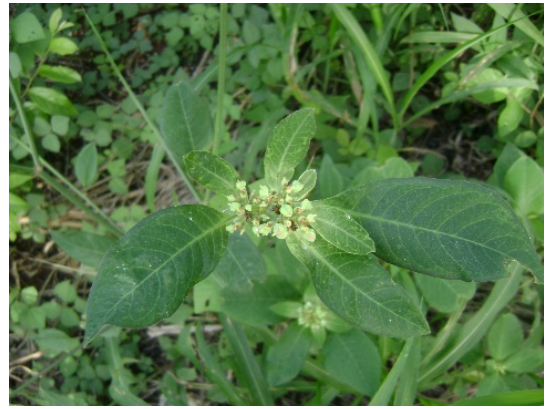

C



D

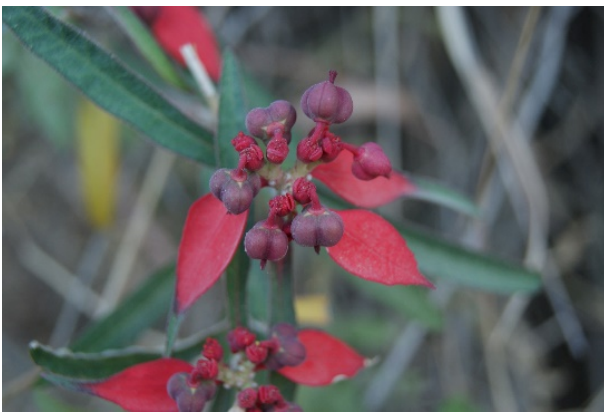

$\mathbf{E}$

Fig. 10. Especies del Euphorbia sect. Poinsettia con potencial ornamental. A. E. cornastra. B. E. pulcherrima. C. E. heterophylla. D. E. cyathophora y E. E. strigosa. 


\section{DISCUSIÓN}

\section{Distribución geográfica}

La distribución de las especies analizadas en este trabajo presenta diferencias con lo reportado por (Mayfield, 1997). De acuerdo a este autor Euphorbia heterophylla se distribuye en 24 estados de México; sin embargo, en el presente estudio fue posible localizarla en otros cinco estados más: Aguascalientes, Campeche, Durango, Tlaxcala y Zacatecas. El mismo autor documentó que E. cyathophora se distribuye en 23 estados incluyendo la Ciudad de México, en la cual no fue posible localizar algún ejemplar. No obstante, en el presente estudio se ubicaron ejemplares en otros seis estados: Baja California Sur, Nuevo León, Querétaro, Tabasco y Durango. En los herbarios de México no se localizan ejemplares del estado de Sinaloa; sin embargo, se localizó un ejemplar en los herbarios LL y TEX.

La información obtenida en este trabajo amplía la distribución de Euphorbia radians, a seis estados más: Aguascalientes, Michoacán, Morelos, Querétaro, Veracruz y Zacatecas. E. pulcherrima se distribuye en todos los estados que menciona Mayfield (1997), excepto en Durango, en el cual no fue posible encontrar algún ejemplar. La distribución de poblaciones silvestres coincide con la reportada por Trejo et al. (2012). Cabe mencionar que se localizaron ejemplares de herbario que consignan como silvestres; sin embargo, como los puntos de colecta se localizan cerca de poblados es posible que se trate de plantas cultivadas en jardines. En los recorridos de campo realizados se observaron plantas que se han asilvestrado, generalmente son manchones cerca de los poblados, plantas en cultivos o en construcciones abandonadas.

Euphorbia colorata ha sido reportada en el estado de Zacatecas; sin embargo, en este estudio no se encontró algún ejemplar que corresponda a dicho estado. (Mayfield, 1997) menciona que $E$. restiaceae se distribuye en los estados de Durango y Nayarit, no obstante, en este estudio se encontró que se distribuye en Nayarit y Jalisco. Para el caso de E. strigosa, (Mayfield, 1997) reporta que se distribuye en cinco estados: Colima, Jalisco, Nayarit, Sinaloa y Sonora. Sin embargo, en este trabajo se adiciona Durango y también se localizó un ejemplar en el límite entre Sonora y Chihuahua en el herbario de ARIZ, así como un segundo ejemplar perteneciente a la Sierra de Charuco, el cual no pudo ser georreferenciado. En el herbario de MO hay una ejemplar de Zacatecas que se ubica entre los límites de Zacatecas y Jalisco. Por su parte, Valdez-Hernández et al. (2018) afirmaron que E. strigosa se distribuye en San Luis Potosí y Veracruz, pero no se encontraron ejemplares de herbario que corroboren dicha información.

Cabe mencionar que ejemplares localizados en herbarios de Estados Unidos no se localizaron en los herbarios de México, por lo que sería importante realizar exploraciones botánicas en los estados donde sólo se han colectado uno o dos ejemplares con objeto de corroborar la distribución indicada en los mismos. En la página de Naturalista se han realizado observaciones de algunas especies en estados donde no se registraron ejemplares de herbario: E. cyathophora en Guanajuato, E. hormorrhiza en Nuevo León, E. pumicicola en Sinaloa y E. radians en Tlaxcala, por lo que sería conveniente realizar exploraciones y corroborar la identificación y distribución de las especies en dichos estados.

Existen patrones de distribución en función del hábito de crecimiento, las plantas anuales se distribuyen en casi todo el territorio mientras que las hierbas perennes y arbustos presentan distribución restringida, lo cual coincide con lo señalado por Vargas-Amado et al. (2013), quienes estudiando el género Cosmos determinaron que los hábitos de crecimiento mostraron patrones de distribución geográfica diferentes y observaron que las hierbas perennes tienen una distribución más restringida en comparación con las hierbas anuales. Esto no ocurrió con $E$. pumicicola, ya que a pesar de ser una hierba anual presenta distribución restringida, por lo que se sugiere realizar exploraciones que permitan determinar si se localiza en otros estados. Este patrón tampoco se observó en E. pulcherrima, que a pesar de presentar plantas con hábito arbustivo o como árboles pequeños tiene una distribución amplia en comparación con las otras hierbas perennes y arbustos evaluados en este trabajo. 
Otro rasgo importante a resaltar es el endemismo, la mayoría de las especies son endémicas de nuestro país, por lo que con base en la propuesta de Rzedowski (1991) se podría considerar que Euphorbia radians es endémica en Megaméxico I y E. pulcherrima en Megaméxico II, por lo que podríamos considerar que, a excepción de las hierbas anuales, todas las especies estudiadas en este trabajo son endémicas. A pesar de que la mayoría de las especies tienen distribución restringida y son endémicas de México y de que las poblaciones observadas muestran algún grado de perturbación, no se encontraron dentro de alguna categoría de la NOM-059SEMARNAT-2010 y tampoco en los apéndices del CITES (Convención sobre el Comercio Internacional de Especies Amenazadas de Fauna y Flora Silvestres).

En el presente año, la nochebuena se integra a la lista roja de la Unión Internacional para la Conservación de la Naturaleza (IUCN) bajo la categoría de preocupación menor, es decir, es considerada un taxon abundante y de amplia distribución y con una tendencia poblacional estable. Eso significa que no cumple con ninguno de los criterios que definen las categorías en peligro crítico, en peligro, vulnerable o casi amenazado. Sin embargo, durante los recorridos realizados en el presente estudio se observaron poblaciones con algún grado de perturbación debido a la deforestación y contaminación de sus hábitats. Por lo que se sugiere que se integren a programas de conservación in situ o ex situ, tomando como base lo documentado por Trejo et al. (2012), quienes mostraron que poblaciones de nochebuena se ubican dentro de áreas naturales protegidas, con alta fragmentación y en sus observaciones de campo hablan de la posible extinción de cuatro poblaciones de E. pulcherrima, por lo que consideran importante la conservación de las poblaciones silvestres.

\section{Potencial de uso ornamental}

Euphorbia cyathophora en algunos países se cultiva en jardines e incluso se han realizado estudios para su propagación in vitro (Rodríguez-Elizalde et al., 2019), sin embargo, en este estudio se evaluó que a pesar de poseer valores estéticos presenta comportamiento de maleza bajo cultivo. Euphorbia heterophylla no presenta valores estéticos y se esparce fácilmente hacia otras macetas. A pesar de que ambas se encuentran en los listados de plantas con potencial ornamental (Corona \& Chimal, 2006), tienen amplia distribución y fácil propagación se recomienda mantenerlas dentro de las colecciones porque podrían ser de utilidad para conferir alguna característica a otras especies, por ejemplo, resistencia a plagas y enfermedades.

Por su parte, Euphorbia cornastra y E. strigosa presentan el potencial de ser usadas como plantas de ornato, bajo la condición de que se logre establecer un método efectivo de propagación para contar con un número considerable de plantas y se pueda dar paso a la siguiente fase que consiste en elegir técnicas de mejoramiento genético y realizar experimentos enfocados a su producción. Euphorbia cornastra ha sido utilizada en Estados Unidos para obtener un híbrido con E. pulcherrima, además se ha establecido que tiene gran potencial como nuevo cultivo ornamental en maceta (Le Duc \& Albrecht, 1996). En el caso de la nochebuena ya existen grupos de trabajo enfocados en generar variedades mexicanas utilizando plantas silvestres y cultivadas (Jaime Canul-Ku et al., 2018), sin embargo, se debe resaltar el potencial que tiene para producir plantas de exterior que conserven su hábito arbustivo y evaluar su uso como flor de corte.

El potencial ornamental en especies silvestres se ha definido con base en características morfológicas que se consideran estéticas, (Corona \& Chimal, 2006), no obstante, el concepto de belleza es subjetivo y en este trabajo se observó que algunas especies aun cuando presentan características morfológicas atractivas no se adaptan fácilmente a cultivo, por lo que es importante conjuntar ambos aspectos. Debido a la diversidad de la familia Euphorbiaceae y del género Euphorbia se recomienda investigar otras especies que además de poseer valores estéticos se adapten bajo cultivo, habiten en lugares secos, tengan tolerancia al frío y resistencia a enfermedades, buscando que el manejo agronómico requiera menos agua y menos uso de agroquímicos. Se sugiere evaluar el potencial de otras especies incluidas dentro del subgénero Chamaesyce, se podría comenzar con especies cuya distribución se restringe al nuevo mundo de 
la sect. Anisophyllum y sect. Alectoroctonum, que son las secciones más cercanas a la sect. Poinsettia.

\section{CONCLUSIONES}

Las especies estudiadas en el presente trabajo se distribuyen en el continente americano, la mayoría se localiza en estados que se ubican en la vertiente del Pacífico y son endémicas de México, crecen principalmente en suelos tipo regosol, litosol y vertisol y en climas cálidos y templados subhúmedos. Entre las especies evaluadas, Euphorbia cornastra y E. strigosa tienen potencial para ser usadas como plantas ornamentales a reserva de que se encuentren métodos efectivos de propagación y se logren establecer experimentos enfocados a su cultivo. Un área de oportunidad es el mejoramiento de E. pulcherrima como planta de exterior. Se recomienda buscar otras especies de euforbias que se puedan propagar con mayor facilidad que las evaluadas y establecerse bajo cultivo en menor tiempo para que se pueda concluir a la brevedad el proceso de investigación y así pasar a la fase de comercialización.

\section{Agradecimientos}

Se agradece a la Red de Nochebuena por el financiamiento de este trabajo, a los curadores de los herbarios por el acceso y facilidades otorgadas. Al maestro en ciencias Amando Espinosa Flores encargado del Banco de Germoplasma de la UACh, al doctor Víctor Steinmann por su amabilidad y disponibilidad para resolver dudas. A los revisores anónimos por sus sugerencias que mejoraron considerablemente este trabajo.

\section{LITERATURA CITADA}

APG IV. (2016). An update of the Angiosperm Phylogeny Group classification for the orders and families of flowering plants: APG IV. Botanical Journal of the Linnean Society, 181(1), 1-20. https://doi.org/10.1111/boj.12385.

Armitage, A. M. (1986). Evolution of new floricultural crops: a system approach. HortScience, 21, 9-11.

Armitage, A. M. (1987). What is a new crop. Acta Horticulturae, 205, 1-2.

Baillon, M. H. (1987). Étude Générale du groupe des Euphorbiacées. Librairie De Victor Masson, Paris.

Bossier, E. (1862). Euphorbiaceae. In Prodromus Systematis Universalis Regni Vegetabilis. Parte 15 (2). Victoris Masson et Filli, Paris.

Canul-Ku, J., García-Pérez, F., Ramírez-Rojas, S., \& Osuna-Canizalez, F. de J. (2010). Estrategias para el mejoramiento genético de Nochebuena (Euphorbia pulcherrima Willd. ex Klotzsch). Investigación Agropecuaria, 7(1), 44-54.

Canul-Ku, Jaime, García-Pérez, F., Barrios-Gómez, E. J., \& Rangel-Estrada, S. E. (2018). Formación de híbridos clonales en nochebuena (Euphorbia pulcherrima Willd . ex Klotzsch ). Revista Fitotecnia Mexicana, 41(3), 311-316.

Canul-Ku, Jaime, García-Pérez, F., Barrios-Gómez, E. J., \& Rangel-Estrada, S. E. (2019). Juanita, a new variety of pointsettia for indoors derived by Mutagenesis. Revista Fitotecnia Mexicana, 42(2), 191-192. https://doi.org/10.35196/rfm.2019.2.191-192.

Canul Ku, J., García Pérez, F., Barrios Gómez, E. J., Rangel Estrada, S. E., Ramírez Rojas, S. G., \& Osuna Canizalez, F. D. J. (2017). Alondra: nuevo híbrido de nochebuena para interiores. Revista Mexicana de Ciencias Agrícolas, 8(5), 1203. https://doi.org/10.29312/remexca.v8i5.119.

Cázarez-Prado, M. (2013). Propagación y conservación in vitro de Euphorbia strigosa Hook. \& Arn. (tesis de maestría). Universidad Autónoma del Estado de Morelos, México. 
Cerón-Venegas, I. (2006). Colecta y caracterización de especies del género Euphorbia del Estado de Nayarit (tesis de pregrado). Universidad Autónoma Chapingo, México.

Chimal, A. \& Corona, V. (2003). Arbustos mexicanos con potencial ornamental. In J. Mejía \& A. Espinosa (Eds.), Plantas nativas de México con potencial ornamental: análisis y perspectivas (pp. 31-51). Universidad Autónoma Chapingo, México.

Colinas-León, M. T., Espinosa-Flores, A., Mejía-Muñoz, J., Rodríguez-Elizalde, M. A., PérezNicolás, M., \& Alia-Tejacal, I. (2015). Cultivars of Euphorbia pulcherrima from Mexico. Acta Horticulturae, 1104, 487-490.

CONABIO. (2008). Georreferenciación de localidades de colecciones biológicas. Manual de procedimientos. Comisión Nacional para el Conocimiento y Uso de la Biodiversidad, México.

Corona, V. \& Chimal, A. (2006). Plantas mexicanas con potencial ornamental. Universidad Autónoma Metropolitana, Unidad Xochimilco.

Dressler, R. L. (1962). A synopsis of Poinsettia (Euphorbiaceae). Annals of the Missouri Botanical Garden, 48, 329-341.

ESRI. (2019). ArcMap 10.8. Environmental Systems Research Institute, Inc. CA. Estados Unidos.

Flores-Cabrera, G. (2014). Evaluación de la germinación y caracterización morfológica en 24 cruzas de nochebuena (Euphorbia pulcherrima Willd. ex Klotzsch) (tesis de pregrado). Universidad Autónoma Chapingo, México.

Galindo-García, D. V., Alia-Tejacal, I., Núñez-Colin, C. A., Andrade-Rodríguez, M., CanulKu, J., Colinas-León, M. T., \& Sainz-Aispuro, M. de J. (2019). Genetic diversity of sun poinsettia (Euphorbia spp.) in Morelos, Mexico, with RAPD molecular markers. Revista Chapingo, Serie Horticultura, 25(2), 113-127. https://doi.org/10.5154/r.rchsh.2018.06.012

Galindo-García, D. V., Andrade-Rodríguez, M., Colinas-León, M. T., \& Canul-Ku, J. (2012). Producción de nochebuena de sol en Morelos, México * Sun-poinsettia production in Morelos, Mexico. Revista Mexicana de Ciencias Agrícolas, 3(4), 751-763.

GBIF. (2018). The Global Biodiversity Information Facility. Recuperado de http://www.gbif.org/what-is-gbif. Fecha de consulta: Febrero, 2018.

González-Fierro, P. H. (2007). Germinación y desarrollo fenológico de nochebuenas silvestres (Euphorbia pulcherrima Willd. ex Klotzsch) (tesis de pregrado). Universidad Autónoma Chapingo, México.

Graham, R. (1836). Description of several new or rare plants which have latery flowered in the Neighbourhood of Edinburth, chiefly in the Royal Botanic Garden. Edinburgh New Philosophical Journal, 20, 412-413.

Guadarrama-Martínez, N., Rubí-Arriaga, M., González-Huerta, A., Vázquez-García, L. M., Martínez-de la Cruz, I., López-Sandoval, J. A., \& Hernández-Flores, G. V. (2012). Inventario de árboles y arbustos con potencial ornamental en el sureste del estado de México. Phyton, 81, 221-228.

Hernández-Carrillo, N. (2014). Caracterización del desarrollo de Euphorbia cornastra (Dressler) Radcl. Sm. (tesis de maestría). Universidad Autónoma Chapingo, México.

Horn, J. W., Van Ee, B. W., Morawetz, J. J., Riina, R., Steinmann, V. W., Berry, P. E., \& Wurdack, K. J. (2012). Phylogenetics and the evolution of major structural characters in the giant genus Euphorbia L. (Euphorbiaceae). Molecular Phylogenetics and Evolution, 63, 305-326. https://doi.org/10.1016/j.ympev.2011.12.022.

House, H. D. (1924). Annotated list of the ferns and flowering plants of New York State. New York State Museum Bulletin, 245, 470-473.

Huft, M. J. (1984). A review of Euphorbia (Euphorbiaceae) in Baja California. Missouri Botanical Garden Press, 71, 1021-1027.

Hurrel, J. A. (2016). Ornamental Plants. In U. P. Albuquerque \& R. R. N. Alves (Eds.), Introduction to Ethnobiology (pp. 171-176). https://doi.org/10.1007/978-3-319-28155-1.

INEGI. (2008). Unidades climáticas. Conjunto de datos vectoriales escala: 1:1 000000. Formato digital. Instituto Nacional de Estadística y Geografía, México. Recuperado de http://www.inegi.org.mx/app/biblioteca/ficha.html?upc=70282567568. Fecha de 
consulta: Abril, 2020.

INEGI. (2014). Perfiles de suelos, Estados Unidos Mexicanos. Conjunto de datos vectoriales escala 1:1 000 000. Formato digital. Instituto Nacional de Estadística y Geografía, México. Recuperado de http://internet.contenidos.inegi.org.mx/contenidos/Productos/prod serv/contenidos/esp anol/bvinegi/productos/geografia/tematicas/PERFIL_SUELOS/702825267636_s.zip. Fecha de consulta: Abril, 2020.

IPNI. (2019). International Plant Names Index. Recuperado de http://www.ipni.org. Fecha de consulta: Agosto, 2019.

Le Duc, A., \& Albrecht, M. L. (1996). Dogwood Poinsettia Euphorbia cornastra (Dressler) A. Radcliffe-Smith, a new floral pot crop. HortScience, 31(3), 472.

Li, X. X., \& Zhou, Z. K. (2005). Endemic wild ornamental plants from northwestern Yunnan, China. HortScience, 40(6), 1612-1619. https://doi.org/10.21273/hortsci.40.6.1612

Martínez-Gordillo, M., Jiménez-Ramírez, J., Cruz-Durán, R., Juárez-Arriaga, E., García, R., Cervantes, A., \& Mejía-Hernández, R. (2002). Los géneros de la familia Euphorbiaceae en México (parte A). Anales Del Instituto de Biología. Serie Botánica, 73(2), 155-281.

Martínez-Villegas, Y. M., Andrade-Rodríguez, M., Colinas-León, M. T., Villegas-Torres, Ó. G., Castillo-Gutiérrez, A., \& Alia-Tejacal, I. (2015). Efecto de las sales inorgánicas del medio de cultivo en el crecimiento de pascuita (Euphorbia leucocephala Lotsy). Revista Fitotecnia Mexicana, 38(4), 369-374.

Martínez, M., Steinmann, V., Jiménez, J., Cervantes, A., Ramírez, Y., \& Ramírez, A. (2008). Euforbiáceas ( Dicotiledóneas ). In S. Ocegueda \& J. Llorente-Bousquets (Eds.), Catálogo taxonómico de especies de México, en Capital Natural de México: Vol. I (pp. 1-58).

Mayfield, M. H. (1997). A Systematic Treatment of Euphorbia Sugenus Poinsettia (Euphorbiaceae) (doctoral thesis). Uniersity of Texas at Austin, Estados Unidos.

Pérez-Nicolás, M., \& Fernández-Nava, R. (2007). Plantas del Estado de Querétaro, México con potencial para uso ornamental. Polibotánica, 24, 83-115.

REMIB. (2015). Red Mundial de Información sobre Biodiversidad. Recuperado de http://www.conabio.gob.mx/remib/doctos/remibnodoshdb.html?. Fecha de consulta: Febrero, 2018.

Rendón-Correa, A., \& Fernández-Nava, R. (2007). Plantas con potencial uso ornamental del estado de Morelos, México. Polibotánica, 23, 121-165.

Rodríguez-Elizalde, M., Ferrera-Cerrato, R., Vargas-Hernández, M., Colinas-León, M. T., Manzo-González, A., \& Alarcón, A. (2019). Towards the micropropagation of Euphorbia cyathophora Murray: A wild plant species with medicinal and ornamental potential. Ciencia Rural, 49(9), e20180716. https://doi.org/10.1590/0103$8478 \mathrm{cr} 20180716$

Rzedowski, J. (1991). El endemismo en la flora fanerogámica mexicana: una apreciación analítica preliminar. Acta Botanica Mexicana, 15, 47-64.

Rzedowski, J. (1995). Aspectos de las plantas ornamentales mexicanas. Revista Chapingo, Serie Horticultura, 1(3), 5-7.

Steinmann, V. W. (2002). Diversidad y endemismo de la familia Euphorbiaceae en México. Acta Botanica Mexicana, 61, 61-93. https://doi.org/10.21829/abm61.2002.909

Trejo-Hernández, L., Olson-Zúnica, M. E., \& Bye-Boettler, R. A. (2015). Datos históricos y diversidad genética de las nochebuenas (Euphorbia pulcherrima) del Distrito Federal, México. Revista Mexicana de Biodiversidad, 86, 478-485. https://doi.org/10.1016/j.rmb.2015.04.033.

Trejo, L., Feria-Arroyo, T. P., Olsen, K. M., Eguiarte, L. E., Arroyo, B., Gruhn, J. A., \& Olson, M. E. (2012). Poinsettia's wild ancestor in the Mexican dry tropics: Historical, genetic, and environmental evidence. American Journal of Botany, 99(7), 1146-1157. https://doi.org/10.3732/ajb.1200072.

Tropicos. (2018). Missouri Botanical Garden. Recuperado de http://tropicos.org. Fecha de consulta: Febrero, 2018. 
Recibido:

11/noviembre/2019

Aceptado:

23/junio/2020

UNAM. (2019). Datos abiertos UNAM Colecciones Universitarias. Herbario Nacional de México, Plantas Vasculares. Departamento de Botánica, Instituto de Biología (IBUNAM). Recuperado de http://datosabiertos.unam.mx. Fecha de consulta: Enero, 2019.

Valdez-Hernández, E. F., Flores-Vilchez, F., Pedraza-Santos, M. E., Colinas-León, M. T., Ramírez-Guerrero, L. G., Martínez-Cárdenas, L., \& García-Díaz, R. F. (2018). Distribución de Euphorbia strigosa Hook and Arn planta nativa de México con potencial ornamental. Revista Bio Ciencias, 5(1), e303. https://doi.org/10.15741/revbio.05.2018.02.

Valdez-Hernández, Edna F., Juárez-López, P., Pedraza-Santos, M. E., Zamora-Becerra, B. L., \& Martínez-Cárdenas, L. (2017). Descripción de semillas silvestres de Euphorbia strigosa Hook and Arn del estado de Nayarit, México. Temas Agrarios, 22(1), 41-51. https://doi.org/10.21897/rta.v22i1.914.

Vargas-Amado, G., Castro-Castro, A., Harker, M., Villaseñor, J. L., Ortiz, E., \& Rodríguez, A. (2013). Geographic distribution and richness of the genus Cosmos (Asteraceae: Coreopsideae). Revista Mexicana de Biodiversidad, 84, 536-555. https://doi.org/10.7550/rmb.31481.

Villaseñor, J. L. (2016). Checklist of the native vascular plants of Mexico. Revista Mexicana de Biodiversidad, 87, 559-902. https://doi.org/10.1016/j.rmb.2016.06.017.

Webster, G. L. (1994). Synopsis of the Genera and Suprageneric Taxa of Euphorbiaceae. Annals of the Missouri Botanical Garden, 81, 33-144. https://doi.org/10.2307/2399909.

Yang, Y., Riina, R., Morawetz, J. J., Haevermans, T., Aubriot, X., \& Berry, P. E. (2012). Molecular phylogenetics and classification of Euphorbia subgenus Chamaesyce (Euphorbiaceae). Taxon, 61(4), 764-789.

Apéndice 1. Clasificaciones que hacen referencia a la sección Poinsettia.

\begin{tabular}{llll}
\hline $\begin{array}{l}\text { Euphorbia } \text { sect. } \\
\text { Poinsettia } \\
\text { Bossier, } 1862\end{array}$ & $\begin{array}{l}\text { Género Poinsettia } \\
\text { Dressler (1962) }\end{array}$ & $\begin{array}{l}\text { Euphorbia subg. Poinsettia } \\
\text { Mayfield, 1997 }\end{array}$ & $\begin{array}{l}\text { Euphorbia } \text { sect. Poinsettia } \\
\text { (Graham) Baill. } \\
\text { Yang } \text { et al., 2012 }\end{array}$ \\
\hline $\begin{array}{l}\text { E. pulcherrima } \\
\text { E. dentata }\end{array}$ & $\begin{array}{l}\text { P. pulcherrima } \\
\text { P. radians }\end{array}$ & $\begin{array}{l}\text { Primer grupo: Euphorbia } \\
\text { dentata alliance }\end{array}$ & $\begin{array}{l}\text { Euphorbia } \text { subsect. Lacerae } \\
\text { subsect. nov. }\end{array}$ \\
E. geniculata & P. restiaceae & E. pentadactyla & \\
E. heterophylla & P. colorata & E. cuphosperma & \\
E. cuphosperma & P. strigosa & E. davidii & \\
E. barbellata & P. coccinea & E. dentata & \\
E. colorata & P. pentadactyla & E. schiedeana & Euphorbia subsect. Erianthae \\
E. eriantha & P. inornate & E. subilsae sp. nov. & \\
E. strigosa & P. heterophylla & E. tubadenia & \\
E. radians & $P$. cyathophora & & \\
E. restiacea & P. dentata & &
\end{tabular}

Euphorbia subsect. Exstipulatae subsect. nov. 
Segundo grupo: Subgénero

Poinsettia sensu stricto

E. tiarata sp. nov.

E. heterophylla

E. kurtzii

E. abscondita

E. elliptica

E. cyathophora

E. richardsonii

E. hormorrhiza

E. pinetorum

E. colorata

E. strigosa

E. radians var. radians

E. radians var. stormiae

E. restiacea

E. pulcherrima

E. cornastra

E. pumicicola

E. zonosperma
Euphorbia subsect. Stormieae

E. chersonesa

E. colorata

E. cornastra

E. cuphosperma

E. cyathophora

E. dentata

E. heterophylla

E. hormorrhiza

E. pentadactyla

E. pinetorum

E. pulcherrima

E. pumicicola

E. radians

E. strigosa

E. zonosperma 
Apéndice 2. Nombres comunes, usos y floración de especies de Euphorbia sect. Poinsettia subsect. Stormiae.

\begin{tabular}{|c|c|c|c|}
\hline Especie & Nombres comunes & Usos & $\begin{array}{c}\text { Floración } \\
\text { (colectas con flores) }\end{array}$ \\
\hline E. colorata & $\begin{array}{l}\text { contrahierba, contrayerba (Sin, Son, Dgo), toxkolh bhich } \\
\text { (Dgo) }\end{array}$ & Medicinal & Marzo a diciembre \\
\hline E. cornastra & -- & -- & Junio, julio y agosto \\
\hline E. cyathophora & $\begin{array}{l}\text { pascua silvestre (Mex), pascuarilla (Gro), nochebuena } \\
\text { silvestre (Jal), Tzutz zantun wamal-tzeltal (Chis), Hoboon } \\
\text { kaak' Hobon xiu, xiax-halal-ch, xuechiu (Yuc, Camp), hierba } \\
\text { del alormo (Nay) }\end{array}$ & $\begin{array}{l}\text { Medicinal } \\
\text { y Forrajero }\end{array}$ & Durante todo el año \\
\hline E. heterophylla & $\begin{array}{l}\text { xhobon kaak (Yuc, Qro), lechosa, lechosilla, lecherilla, } \\
\text { lechoso (Nay, Ver, Jal, Mich, Tab), hierba de la araña (Zac, } \\
\text { Ver), pascuilla, pascuarilla (Mich, Gro), antizl chu' tzoltzil, } \\
\text { ka'abak nich wamal, pameyat Tzeltal (Chis), pune yaquey- } \\
\text { zoque (Oax), calderona, catalina, drago (Jal), contrahierba } \\
\text { (Dur), nochebuena silvestre (Gro) }\end{array}$ & $\begin{array}{l}\text { Medicinal } \\
\text { y Forrajero }\end{array}$ & Durante todo el año \\
\hline E. hormorrhiza & -- & -- & Octubre \\
\hline E. pulcherrima & $\begin{array}{l}\text { catarina (Oax), Catalina (Sin), pascua o flor de pascua (Jal), } \\
\text { cuetlaxochitl o pascuaxochitl (Pue), chakmaché, flor de } \\
\text { pastor (Chis) y peseva o bebeta (Ver) }\end{array}$ & $\begin{array}{l}\text { Ornamental } \\
\text { Medicinal }\end{array}$ & Agosto a febrero \\
\hline & & Comestible & \\
\hline E. pumicicola & -- & -- & $\begin{array}{l}\text { Agosto, septiembre y } \\
\text { octubre }\end{array}$ \\
\hline $\begin{array}{l}\text { E. radians var. } \\
\text { radians }\end{array}$ & hierba de la golondrina, matarique (Jal) y colecitas (Mex) & Comestible & Marzo, abril y mayo \\
\hline $\begin{array}{l}\text { E. radians var. } \\
\text { stormiae }\end{array}$ & -- & -- & Enero a mayo \\
\hline E. restiacea & -- & -- & Abril \\
\hline E. strigosa & nochebuena de campo (Nay) & Medicinal & Marzo y abril \\
\hline
\end{tabular}


Apéndice 3. Especies de Euphorbia sect. Poinsettia subsect. Stormiae.

\begin{tabular}{|c|c|c|c|c|c|}
\hline Especie & Tipo de vegetación & Altitud (m) & Estatus & EH & EHBD \\
\hline E. colorata & $\mathrm{BQ}, \mathrm{BPE}, \mathrm{SBC}, \mathrm{P}$ & $500-2400$ & Endémica & 56 & 115 \\
\hline E. cornastra & BQ & $1930-2300$ & Endémica & 10 & 10 \\
\hline E. cyathophora & $\begin{array}{l}\text { OC, M, CH, P, VR, DC, VS de } \\
\text { SBC, SMS, SAP, BQ, BPQ, } \\
\text { BMM }\end{array}$ & $\begin{array}{l}0-2000(2 \\
800)\end{array}$ & Nativa & 298 & 520 \\
\hline E. heterophylla & $\begin{array}{l}\text { OC, C, M, P, VR, DC, VS de } \\
\text { SBC, SBS, BQ, BPQ }\end{array}$ & $0-2600$ & Nativa & 785 & 1287 \\
\hline E. hormorrhiza & E de SBC, BQP & $300-400$ & Endémica & 2 & 3 \\
\hline E. pulcherrima & SBC, SBS, SMS & $280-1500$ & $\begin{array}{l}\text { Endémica } \\
\text { Megamexico } \\
\text { II }\end{array}$ & 206 & 247 \\
\hline E. pumicicola & $\mathrm{M}$ & $60-500$ & Endémica & 1 & 5 \\
\hline $\begin{array}{l}\text { E. radians var. } \\
\text { radians }\end{array}$ & $\begin{array}{l}\mathrm{C}, \mathrm{M}, \mathrm{P}, \mathrm{BQ}, \mathrm{BPQ} \\
\mathrm{VS} \text { de BPE, BE }\end{array}$ & $1350-2600$ & $\begin{array}{l}\text { Endémica } \\
\text { Megamexico } \\
\text { I }\end{array}$ & 115 & 161 \\
\hline $\begin{array}{l}\text { E. radians var. } \\
\text { stormiae }\end{array}$ & $\mathrm{OC}, \mathrm{BQ}, \mathrm{BP}, \mathrm{VS}$ de BQ, BP & $\begin{array}{l}1500-2600 \\
(2800)\end{array}$ & Endémica & 16 & 25 \\
\hline E. restiacea & $\mathrm{BQ}, \mathrm{BPQ}$ & $800-2000$ & Endémica & 7 & 7 \\
\hline E. strigosa & $\begin{array}{l}\mathrm{BQ}, \mathrm{BPQ}, \mathrm{SBC}, \mathrm{SBS}, \mathrm{VS} \\
\text { derivada de las anteriores }\end{array}$ & $\begin{array}{l}(100) 300-2 \\
400\end{array}$ & Endémica & 44 & 91 \\
\hline
\end{tabular}

EH. Ejemplares de herbario. EHBD. Ejemplares de herbario en bases digitales. OC-orillas de camino, C-cultivos, Mmatorrales, P-pastizales, VS-vegetación secundaria, BQ-bosque de Quercus, BP-bosque de Pinus, BPQ-bosque de pino-encino, SBC-selva baja caducifolia, SMS-selva mediana subcaducifolia, SAP, Selva alta perennifolia, BMMbosque mesófilo de montaña, VR-vegetación riparia, DC-dunas costeras. 
Apéndice 4. Características morfológicas de especies de Euphorbia sect. Poinsettia subsect. Stormiae.

\begin{tabular}{|c|c|c|c|c|c|c|}
\hline Especie & $\begin{array}{c}\text { Hábito de } \\
\text { crecimiento }\end{array}$ & Tallos & Raíces & Glándulas & $\begin{array}{l}\text { Color de } \\
\text { brácteas }\end{array}$ & $\begin{array}{c}\text { Número } \\
\text { cromosómico }\end{array}$ \\
\hline E. colorata & Hierba perenne & Glabros & Tuberosa & 1 & $\begin{array}{l}\text { Rojo } \\
\text { brillante }\end{array}$ & Desconocido \\
\hline E. cornastra & Arbusto & Tomentulosos & Fibrosa & $1-2$ & Blanco & $2 n=28$ \\
\hline E. cyathophora & Hierba anual & $\begin{array}{l}\text { Glabros a } \\
\text { esparcidamente } \\
\text { pilosos o } \\
\text { puberulentos }\end{array}$ & Fibrosa & $1-2$ & $\begin{array}{l}\text { Verde con } \\
\text { anaranjado } \\
\text { a rojo en la } \\
\text { base }\end{array}$ & $2 n=28,56$ \\
\hline E. heterophylla & Hierba anual & $\begin{array}{l}\text { Esparcidamente } \\
\text { pilosos a vilosos }\end{array}$ & Fibrosas & $1-2$ & $\begin{array}{l}\text { Verde, a } \\
\text { veces } \\
\text { amarillentas } \\
\text { en la base }\end{array}$ & $2 n=28$ \\
\hline E. hormorrhiza & $\begin{array}{l}\text { Hierba perenne } \\
\text { geófita }\end{array}$ & $\begin{array}{l}\text { Glabros o con pocos } \\
\text { y débiles tricomas } \\
\text { patentes }\end{array}$ & Tuberosa & 1 & $\begin{array}{l}\text { Verde con } \\
\text { la base } \\
\text { anaranjada }\end{array}$ & $2 n=28$ \\
\hline E. pulcherrima & $\begin{array}{l}\text { Árboles } \\
\text { pequeños o } \\
\text { arbustos }\end{array}$ & $\begin{array}{l}\text { Glabros a veces } \\
\text { puberulentos }\end{array}$ & Fibrosas & $1-2$ & $\begin{array}{l}\text { Rojo y } \\
\text { amarillo }\end{array}$ & $2 \mathrm{n}=28,56$ \\
\hline E. pumicicola & Hierba anual & Glabros & Fibrosa & 1 & Verde & Desconocido \\
\hline E. radians & $\begin{array}{l}\text { Hierba perenne } \\
\text { geófita }\end{array}$ & $\begin{array}{l}\text { Glabros a } \\
\text { puberulentos }\end{array}$ & Tuberosa & $1-4(5)$ & $\begin{array}{l}\text { Rosa, } \\
\text { blanco a } \\
\text { rojizo }\end{array}$ & Desconocido \\
\hline $\begin{array}{l}\text { E. radians var. } \\
\text { stormiae }\end{array}$ & $\begin{array}{l}\text { Hierba perenne } \\
\text { geófita }\end{array}$ & Puberulentos & Tuberosa & $5-6$ & $\begin{array}{l}\text { Inconspicua } \\
\mathrm{S}\end{array}$ & Desconocido \\
\hline E. restiacea & $\begin{array}{l}\text { Hierba perenne } \\
\text { geófita }\end{array}$ & Glabros & Tuberosa & $1-2$ & Verde & $2 n=28$ \\
\hline E. strigosa & $\begin{array}{l}\text { Hierba perenne } \\
\text { geófita }\end{array}$ & Pubescentes & Tuberosa & 1 & $\begin{array}{l}\text { Rojo } \\
\text { brillante }\end{array}$ & Desconocido \\
\hline
\end{tabular}

